\title{
Article
}

\section{Copper Oxide/ Functionalized Graphene Hybrid Nanostruc- tures for Room Temperature Gas Sensing Applications}

\author{
Monika Gupta 1,2, *, Huzein Fahmi Hawari 1,2, , Pradeep Kumar 1,2, and Zainal Arif Burhanudin 1,2
}

\begin{abstract}
${ }^{1}$ Department of Electrical and Electronic Engineering, Universiti Teknologi PETRONAS, Seri Iskandar 32610, Perak, Malaysia; pradeep.hitesh@gmail.com (P.K.); zainalarif.burhanudin@utp.edu.my (Z.A.B.)

2 Center of Innovative Nanostructures and Nanodevices (COINN), Universiti Teknologi PETRONAS, Seri Iskandar 32610, Perak, Malaysia

*Correspondence: monika_18000995@utp.edu.my (M.G.); huzeinfahmi.hawari@utp.edu.my (H.F.H.)
\end{abstract}

\begin{abstract}
Oxide semiconductors are conventionally being used as sensing materials in gas sensors, limiting the detection of gases at room temperature (RT). In this work, a hybrid of copper oxide $(\mathrm{CuO})$ with functionalized graphene $(\mathrm{rGO})$ is proposed to achieve gas sensing at $\mathrm{RT}$. The combination of high surface area and presence of many functional groups in $\mathrm{CuO} / \mathrm{rGO}$ hybrid material makes it highly sensitive for gas absorption and desorption. To prepare the hybrid material, a copper oxide suspension synthesized using copper acetate precursor is added to the graphene oxide solution during its reduction using ascorbic acid. Material properties of $\mathrm{CuO} / \mathrm{rGO}$ hybrid and its drop-casted thin films are investigated using Raman, FTIR, SEM, TEM, and four-point probe measurement systems. We find that the hybrid material is enriched with oxygen functional groups (OFGs) and defective sites along with electrical conductivity $(\sim 1.5 \mathrm{k} \Omega / \square)$. The fabricated QCM (quartz crystal microbalance) sensor with a thin layer of $\mathrm{CuO} / \mathrm{rGO}$ hybrid, demonstrates a high sensing response which is twice the response of the rGO-based sensor for $\mathrm{CO}_{2}$ gas at RT. We believe that the $\mathrm{CuO} / \mathrm{rGO}$ hybrid can be highly suitable for existing and future gas sensors used for domestic and industrial safety.
\end{abstract}

Keywords: Sensing materials, $\mathrm{CuO} / \mathrm{rGO}$ hybrid, graphene, QCM, gas sensor, room temperature sensing.

\section{Introduction}

Over the past few decades, the environment is heavily filled with toxic, inflammable, and harmful gases due to the unceasing development of industries and deforestation [1]. The level of these gases is excessively increased in the atmosphere after industrialization. For a safe and healthy environment, the detection of these gases is seriously demanded [2-4]. The need for an effective gas sensor is then extremely important. Numerous gas sensors based on the IDE (interdigitated electrode) [5-7], FET (field-effect transistor) [8,9], MEMS (microelectromechanical systems) [10,11], and QCM [12-14] have been developed. Among them, the QCM-based gas sensor is very effective owing to its attractive features such as accuracy, high sensitivity, and fast response. Also, QCM can be easily integrated with other electronic components $[15,16]$.

In these gas sensors, metal oxide semiconductors such as zinc oxide $(\mathrm{ZnO})$ [17], nickel oxide (NiO) [18], Tin oxide $\left(\mathrm{SnO}_{2}\right)$ [19,20], titanium dioxide $\left(\mathrm{TiO}_{2}\right)$ [21], and copper oxide $(\mathrm{CuO})$ [22] have been fabricated and used as the sensing materials. Among numerous metal oxide semiconductors, $\mathrm{CuO}$ has gained more attention due to its low cost, non-toxicity, facile preparation, and high surface reactivity [23,24]. $\mathrm{CuO}$ is a p-type 
semiconductor and has a narrow bandgap of 1.2-1.9 eV and exhibits excellent properties that have been utilized in a number of applications such as electrode materials for lithium-ion batteries, thin-film electrodes [25], catalysts [21], and gas sensors [26]. However, these $\mathrm{CuO}$ sensing materials need a high temperature to operate, which limits the gas sensing at room temperature.

The incorporation of $\mathrm{CuO}$ with carbon-based materials can be an effective approach to realize reliable gas sensors at room temperature (RT). Carbon-based two-dimensional (2D) material like graphene has outstanding electronic, mechanical, and thermal properties as well as a large surface-to-volume ratio [27-29]. Graphene has been used as a sensing material for RT detection of various gases such as nitrogen dioxide $\left(\mathrm{NO}_{2}\right)$, sulfur dioxide $\left(\mathrm{H}_{2} \mathrm{~S}\right)$, ammonia $\left(\mathrm{NH}_{3}\right)$, and hydrogen $\left(\mathrm{H}_{2}\right)$ [30-33]. But lower electrical conductivity of graphene thin films also limits their sensors to achieve high performance.

Here, we report $\mathrm{CuO} /$ functionalized graphene ( $\mathrm{rGO}$ ) hybrid nanostructure as a promising sensing material for QCM-based sensors for detecting the gas at room temperature. In synthesis, initially, the $\mathrm{CuO}$ nanoparticles (NPs) are prepared using copper acetate as a precursor and then mixed with functionalized graphene oxide solution. The functionalized graphene oxide is achieved using the chemical reduction method by ascorbic acid. The sensing thin films of the synthesized hybrid material are developed by the drop-cast method. The synergistic effect of $\mathrm{CuO}$ and graphene in the hybrid structure can help to achieve the detection of gas at room temperature. The $\mathrm{CuO} / \mathrm{rGO}$ hybrid offers tremendous properties such as enhanced surface reactivity, good electrical conductivity, and large surface area. The $\mathrm{CuO} / \mathrm{rGO}$ hybrid and rGO-based gas sensors are prepared to investigate the $\mathrm{CO}_{2}$ gas sensing at room temperature.

\section{Materials and Methods}

\subsection{Materials}

Graphene oxide (GO) paste (95\%) was purchased from Graphenea (San Sebastian, Spain). Ascorbic acid, ethanol (95\%), and acetone (95\%) were purchased from Sigma Aldrich (St. Louis, MO, USA). Copper acetate (99.9\% purity), hexamethylenetetramine, and isopropyl alcohol (IPA, 99.9\% purity) were bought from R \& M Chemicals. All the chemicals were of analytical grade, no further purification was required for conducting experiments. Deionized (DI) water was used in all preparations.

\subsection{Synthesis of $\mathrm{CuO} /$ Functionalized Graphene Hybrid and Thin Film Development}

The schematic representation of $\mathrm{CuO} / \mathrm{rGO}$ hybrid synthesis is shown in scheme 1 . Initially, $4 \mathrm{~g}$ of copper acetate is added to $60 \mathrm{ml}$ IPA and mixed under the stirring condition at $80^{\circ} \mathrm{C}$ for $20 \mathrm{~min}$. Then, $4 \mathrm{ml}$ of ethanolamine (MEA) is added carefully to the solution and stirred vigorously for $2 \mathrm{~h}$. The prepared solution is left for $24 \mathrm{hrs}$ for aging. The solution is further washed with water and the solid product was separated using centrifugation. Next, $30 \mathrm{ml}$ Graphene oxide $(0.5 \mathrm{mg} / \mathrm{ml})$ was dissolved in $100.00 \mathrm{~mL}$ of DI water and vigorously stirred for $10 \mathrm{~min}$ to form a well-mixed suspension. Then, 100 
mg ascorbic acid (AA) is carefully added to the suspension. Afterward, $0.02 \mathrm{~g}$ of obtained $\mathrm{CuO}$ powder is added immediately to this $\mathrm{GO}$ suspension followed by vigorous stirring at $80{ }^{\circ} \mathrm{C}$ for $4 \mathrm{hrs}$. The suspension is further washed with ethanol and water (1:1 ratio). The solid product was later collected after the centrifugation followed by drying in the oven at $60^{\circ} \mathrm{C}$.

For comparison, the rGO is also synthesized using our previous work [34]. In brief, $15 \mathrm{ml}$ of GO was added in $30 \mathrm{ml}$ of DI water followed by ultrasonication for $15 \mathrm{~min}$ to acquire a uniform aqueous dispersion. Then, $100 \mathrm{mg}$ of ascorbic acid was carefully added to the GO suspension, then stirred for $1 \mathrm{~h}$ at $65{ }^{\circ} \mathrm{C}$ under RT environment conditions. The change in color of GO suspension from brown to black suggested the accomplishment of functionalized graphene oxide.
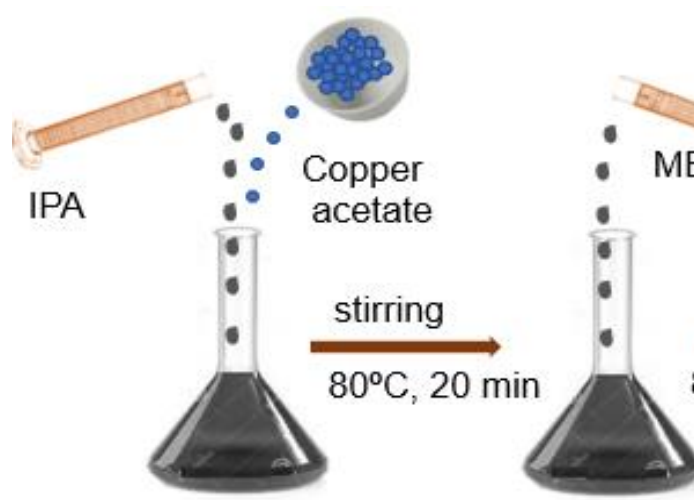

MEA
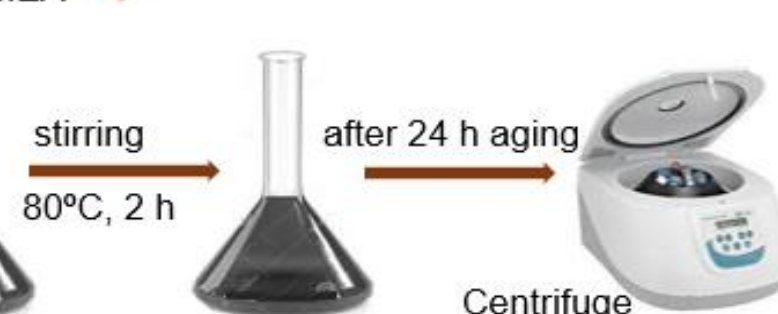

Centrifuge

CuO Suspension

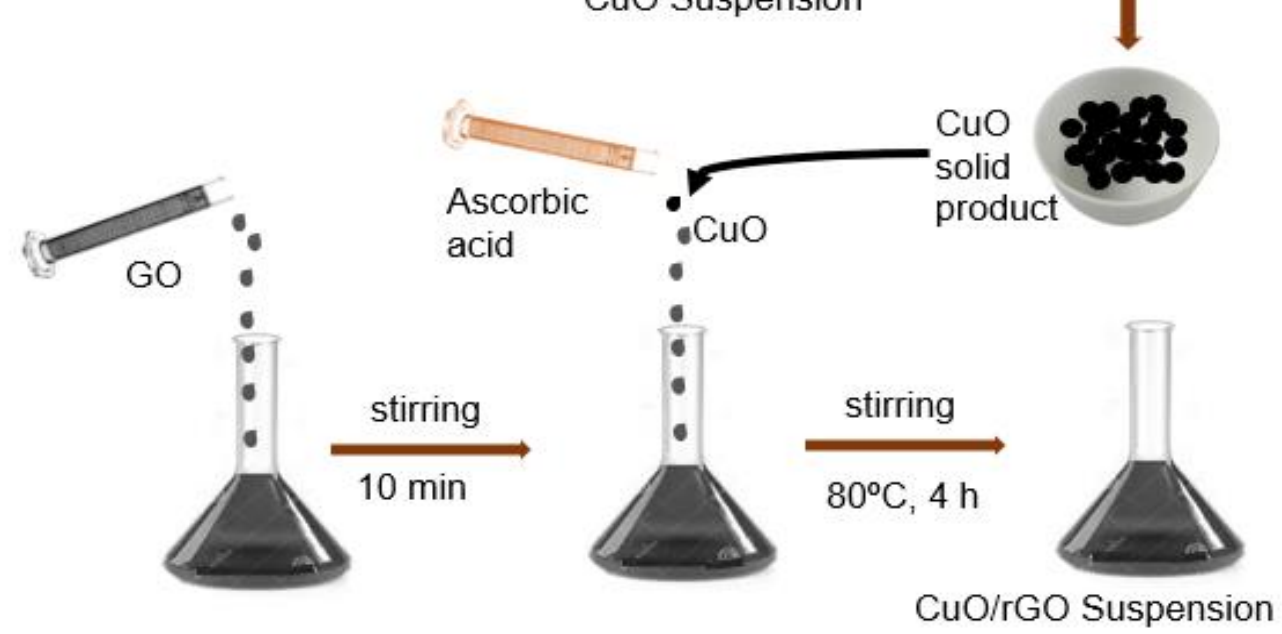

Scheme 1. Schematic representation of $\mathrm{CuO} /$ functionalized graphene (rGO) hybrid synthesis.

The thin films of synthesized $\mathrm{rGO}$ suspensions and $\mathrm{CuO} / \mathrm{rGO}$ hybrid were developed by the drop-casting method. Before thin-film development, the target substrates such as glass and $\mathrm{SiO}_{2}(300 \mathrm{~nm}) / \mathrm{Si}$ were washed in acetone, isopropyl alcohol, DI water, and ethanol using ultrasonication for $15 \mathrm{~min}$ in each solvent.

For sensor fabrication, thin sensing layers of $\mathrm{CuO} / \mathrm{rGO}$ hybrid and $\mathrm{rGO}$ materials were drop casted on a silver $(\mathrm{Ag})$ electrode of $10 \mathrm{MHz}$ QCM (WTL International China, Shenzhen, China). Before that, the QCM resonators were cleaned properly using acetone, isopropyl alcohol (IPA), DI water, and ethanol. A custom-made QCM based gas sensing 
setup is used to get the sensing performance. Before sensing the $\mathrm{rGO}$ and $\mathrm{CuO} / \mathrm{rGO}$ coated QCM were dried at room temperature.

\subsection{Characterization}

The structural and morphological properties of synthesized materials were investigated by Fourier Transform Infrared (FTIR) spectroscopy (Bruker Instruments, model Aquinox 55, Stuttgart), Raman spectroscopy (Horiba Jobin Yvon HR800, Yvon, France, $514 \mathrm{~nm}$ laser excitation) for 200 to $4000 \mathrm{~cm}^{-1}$ spectrum regions, and Transmission electron microscopy (TEM, Zeiss Libra 200FE, Jena, Germany), respectively. The sheet resistance of thin films of prepared materials was measured using a four-point probe measurement system (Lucas Lab 302) with Keithley 2400 source meter.

For gas sensing, $\mathrm{rGO}$ and $\mathrm{CuO} / \mathrm{rGO}$ hybrid coated QCMs were placed inside the QCM holder. In the typical arrangement, the QCM holder is connected to the frequency counter. When gas is pursed to QCM sensors, the analyte gas molecules get absorbed at the material surface and the frequency of QCM gets changed. The frequency counter counts the frequency change. The response and recovery curve can be observed in the attached computer. The sensitivity (S) can be calculated using equation (10) [13],

$$
S=\frac{\text { Frequency shift }(\mathrm{Hz})}{\text { amount of material coated on QCM electrode }(\mu \mathrm{g})}
$$

\section{Results and Discussion}

\subsection{FTIR Analysis}

FTIR spectrum is used to identify the presence of functional groups and chemical compounds. The FTIR spectra of GO, rGO, and $\mathrm{CuO} / \mathrm{rGO}$ hybrid are shown in Figure 1. The FTIR spectrum of GO exhibited strong peaks at $3182 \mathrm{~cm}^{-1}, 1620 \mathrm{~cm}^{-1}$, and $1044 \mathrm{~cm}^{-1}$, attributing to the stretching and deformation of $-\mathrm{OH}$ and $-\mathrm{COOH}$ functional groups as well as the adsorbed and inhibited water molecules to atmospheric moisture $[35,36]$. The peaks at $1724 \mathrm{~cm}^{-1}$ and $1620 \mathrm{~cm}^{-1}$ ascribe to the vibrations of $\mathrm{C}=\mathrm{O}$ stretching and $\mathrm{C}=\mathrm{C}$ alkene groups stretching vibrations. Some other peaks at $1225 \mathrm{~cm}^{-1}$ and $1044 \mathrm{~cm}^{-1}$ were also observed, indicating the presence of $\mathrm{C}-\mathrm{O}$ stretching vibration of epoxy groups and alkoxy group, respectively. Also, in the rGO spectra, weak peaks at $2322 \mathrm{~cm}^{-1}$, and 1391 $\mathrm{cm}^{-1}$ were observed possibly due to the stretching and deformation of $\mathrm{O}-\mathrm{H}$ groups and adsorbed water molecules. The spectrum of $\mathrm{rGO}$ also indicated the presence of the stretching vibration bands for $\mathrm{C}=\mathrm{O}$ at $1717 \mathrm{~cm}^{-1}$ and $\mathrm{C}-\mathrm{O}$ for epoxy and alkoxy at 1219 $\mathrm{cm}^{-1}$ and $1007 \mathrm{~cm}^{-1}$ respectively.

After the formation of the $\mathrm{CuO} / \mathrm{rGO}$ hybrid, the peaks for the functional group were found to be shifted to $3198 \mathrm{~cm}^{-1}$ (hydroxyl), $1570 \mathrm{~cm}^{-1}$ (carboxyl), and $1023 \mathrm{~cm}^{-1}$ (epoxy). This suggests the oxygen functional groups (OFGs) particularly hydroxyls have emerged during the formation of a hybrid nanostructure. However, a weak peak for hydroxyls was found in the rGO nanostructure. In $\mathrm{CuO} / \mathrm{rGO}$ hybrid spectrum, some additional peaks at $560 \mathrm{~cm}^{-1}, 576 \mathrm{~cm}^{-1}$, and $607 \mathrm{~cm}^{-1}$ were also observed, signifying the 
vibrations of the $\mathrm{Cu}-\mathrm{O}$ bond [37]. The presence of these peaks is also attributed to the stabilization of $\mathrm{CuO}$ nanoparticles through the residual OFGs present in $\mathrm{rGO}$ and shows the presence of copper nanoparticles along with rGO [38].

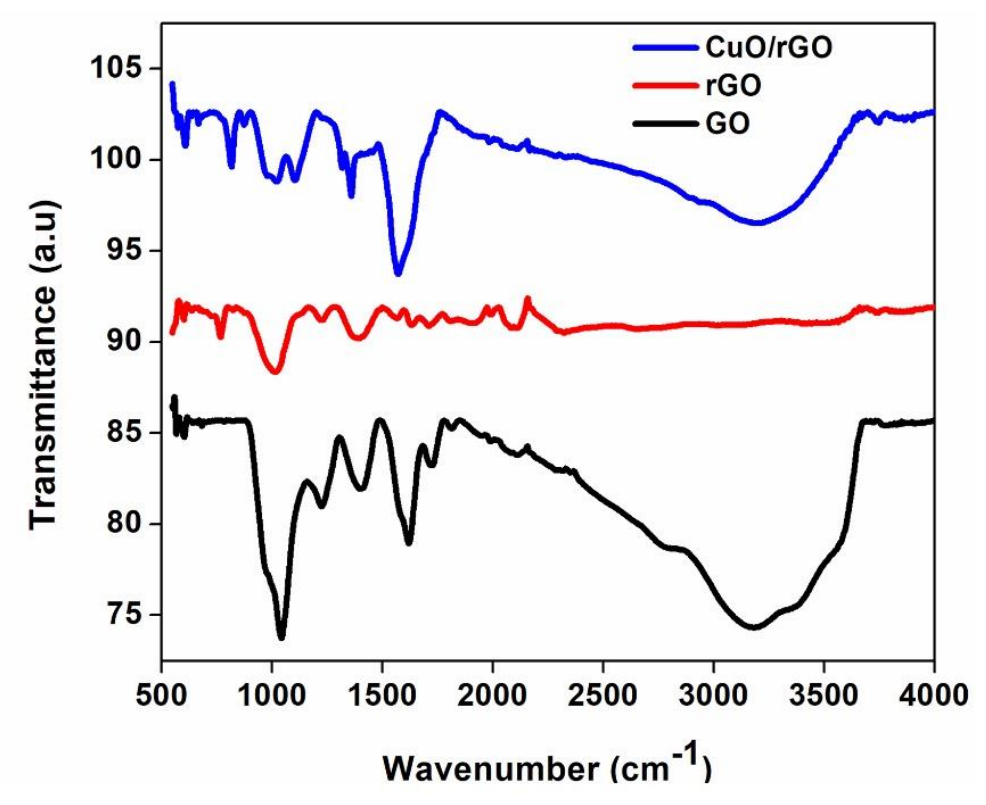

Figure 1. FTIR spectra of GO, rGO, and $\mathrm{CuO} / \mathrm{rGO}$ hybrid.

\subsection{Raman Analysis}

Raman spectrum of $\mathrm{CuO} / \mathrm{rGO}$ hybrid is presented in Figure 2 and compared with spectra of GO, and rGO. The D-peaks for GO, $\mathrm{rGO}$, and $\mathrm{CuO} / \mathrm{rGO}$ hybrid were found at the Raman shift of $\sim 1355 \mathrm{~cm}^{-1}, \sim 1353 \mathrm{~cm}^{-1}$, and $1350 \mathrm{~cm}^{-1}$ respectively. The G-peaks for $\mathrm{GO}, \mathrm{rGO}$, and $\mathrm{CuO} / \mathrm{rGO}$ hybrid were observed at the Raman shift of $\sim 1586 \mathrm{~cm}^{-1}, 1600$ $\mathrm{cm}^{-1}$, and $1594 \mathrm{~cm}^{-1}$ respectively (see figure 3(a)). Typically, the D-peak appears from the defects in $\mathrm{sp}^{3}$ carbon atoms whereas G-peak correlates to the $\mathrm{sp}^{2}$ carbon atoms [39,40]. The prepared $\mathrm{rGO}$ and $\mathrm{CuO} / \mathrm{rGO}$ material inhibit intense D-peak and G-peak, which is attributed to the presence of defects in the graphene layer. The D-peak of rGO and $\mathrm{CuO} / \mathrm{rGO}$ hybrid was observed to be more intense as compared to G-peak, indicating the formation of $\mathrm{sp}^{3}$ graphitic domains and the formation of new defects in the structure during the reduction process [35,41]. The occurrence of two additional peaks at low-frequency Raman shift $\sim 322 \mathrm{~cm}^{-1}$ and $\sim 678 \mathrm{~cm}^{-1}$, indicates the $\mathrm{Ag}_{\mathrm{g}}$ and $\mathrm{Bg}$ modes of 


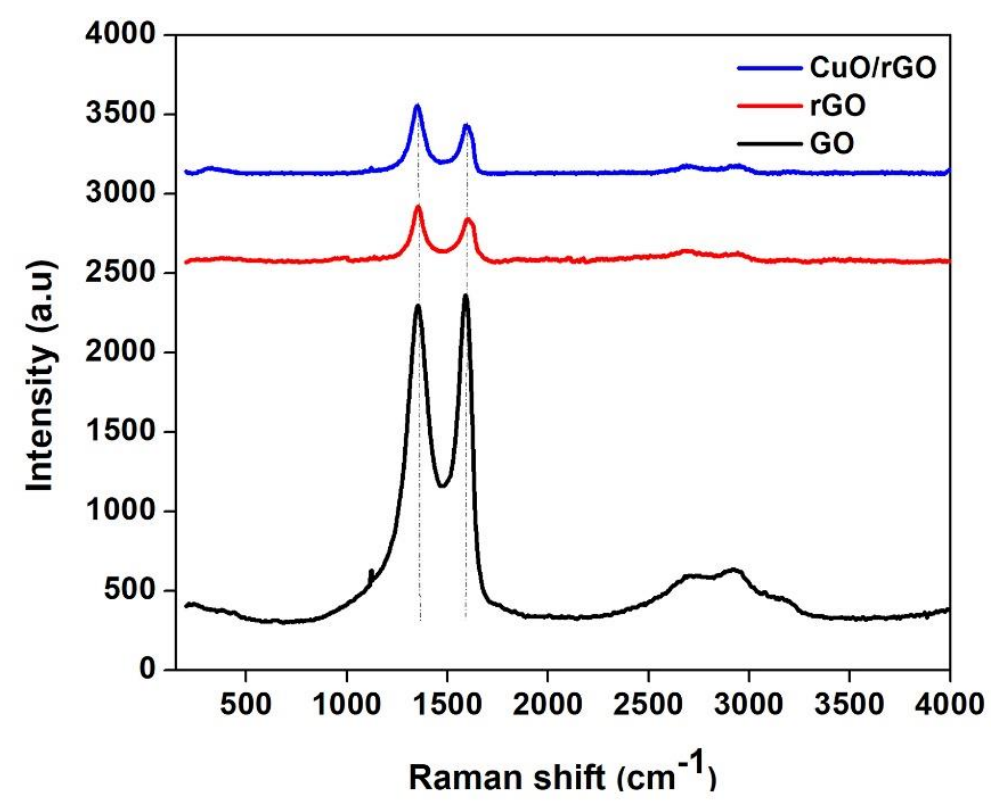

Figure 2. Raman spectra of $\mathrm{CuO} / \mathrm{rGO}$ hybrid, rGO, and GO.

the vibration of $\mathrm{CuO}$ [42]. The 2D peaks were observed at $\sim 2679 \mathrm{~cm}^{-1}, \sim 2689 \mathrm{~cm}^{-1}$, and $\sim 2701 \mathrm{~cm}^{-1}$ for GO, $\mathrm{rGO}$, and $\mathrm{CuO} / \mathrm{rGO}$ hybrid, respectively. The 2D-band also known as $\mathrm{G}^{\prime}$-band, originates from the second-order mode of the D-band [43]. The higher wavenumber position and the lowest peak height of the 2D-band of $\mathrm{CuO} / \mathrm{rGO}$ hybrid suggest the existence of more number of graphene layers as compared to rGO. Moreover, it expresses that the $\mathrm{Cu}$ nanoparticles located between graphene layers, work as a spacer to hinder the agglomeration of graphene layers.
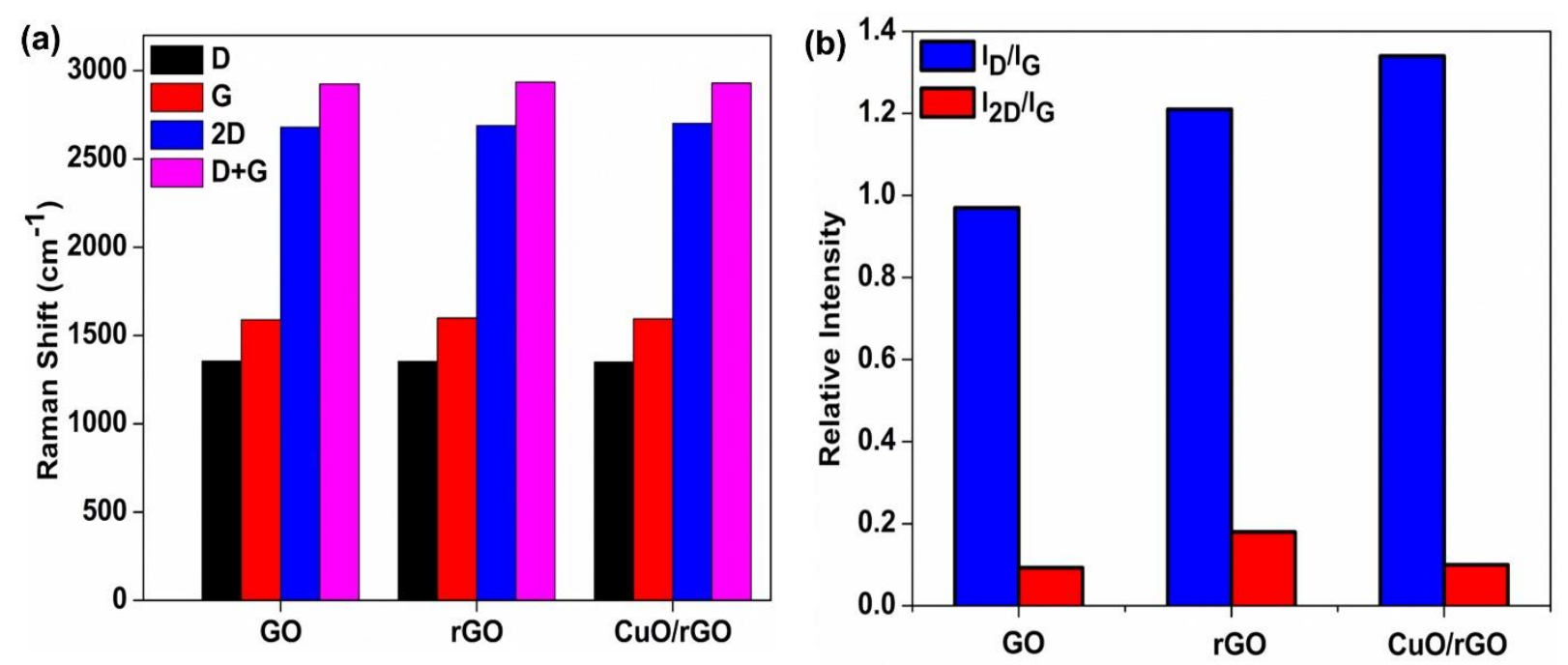

Figure 3. Raman fingerprints- (a) position of D-, G-, 2D-, and D+G - peaks, (b) average values of the parameters $I_{D} / I_{G}, I_{2 D} / I_{G}$ obtained from Raman Spectra of $\mathrm{rGO}$ and $\mathrm{CuO} / \mathrm{rGO}$ hybrid.

The $I_{D} / I_{G}$ intensity ratio (see Figure $3(\mathrm{~b})$ ) was found to be $0.97,1.21$, and 1.35 for GO, $\mathrm{rGO}$, and $\mathrm{CuO} / \mathrm{rGO}$ hybrid, respectively. $I_{D} / I_{G}$ ratio represents the quality or disorder level of graphene. The relatively higher value of $I_{D} / I_{G}$ for $\mathrm{CuO} / \mathrm{rGO}$ hybrid suggests the 
formation of higher graphitic domains (smaller spatial dimensions) [44]. This indicates a decrease in the average size of the $\mathrm{sp}^{2}$ carbon domain upon the reduction of GO. Similar results are also reported by Cheng et al. [45]. The higher intensity of D-peak shows the presence of a large amount of OFGs that are favorable for the physisorption of analyte gas molecules. Reduced graphene oxide consists of graphene layers and OFGs attached at its basal plane and edges [35]. The I2D/IG ratios for GO, rGO, and $\mathrm{CuO} / \mathrm{rGO}$ hybrid were found to be $0.093,0.18$, and 0.13 indicating the presence of few layers of graphene in the $\mathrm{CuO} / \mathrm{rGO}$ hybrid.

The deconvoluted Raman spectra (Lorentz fit) of GO, rGO, and $\mathrm{CuO} / \mathrm{rGO}$ hybrid are shown in Figure 4 . The height of the D band, G band, 2D band, and D+G band is shown in Table 1. It was observed that the intensity of the D-peak of rGO and hybrid were lower than the D-peak of GO, attributing to the presence of fewer OFGs in the rGO and hybrid material. Also, the intensity of G-peak was found to be lower than that of D-peak in rGO and hybrid material, showing that these materials have higher defects [46,47].

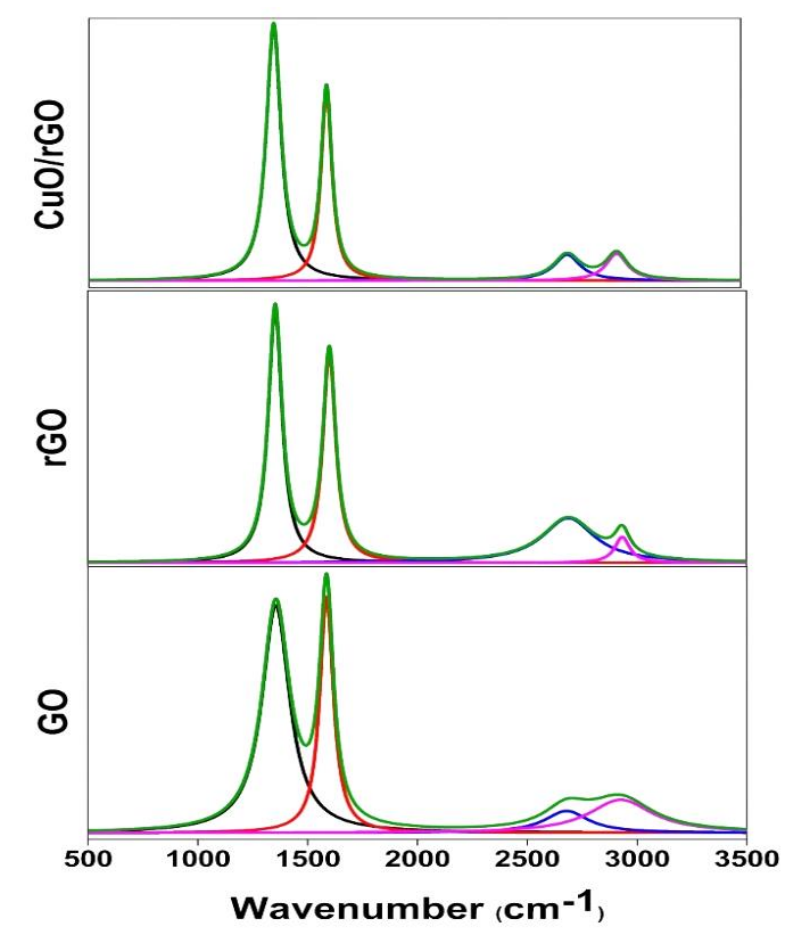

Figure 4. Deconvoluted (Lorentz fit) Raman curve of GO, rGO, and CuO/rGO hybrid.

Full width half maximum (FWHM) values of D- and G-peaks of GO, rGO, and $\mathrm{CuO} / \mathrm{rGO}$ hybrid are shown in Table 2. A lower value of FWHM of D and G band for $\mathrm{CuO} / \mathrm{rGO}$ hybrid $\left(\sim 81 \mathrm{~cm}^{-1}\right.$ and $\left.\sim 60 \mathrm{~cm}^{-1}\right)$ and $\mathrm{rGO}\left(\sim 76 \mathrm{~cm}^{-1}\right.$ and $\left.288 \mathrm{~cm}^{-1}\right)$ in comparison to GO $\left(\sim 153 \mathrm{~cm}^{-1}\right.$ and $\left.75 \mathrm{~cm}^{-1}\right)$ indicates the presence of fewer OFGs in their structures.

Table 1. Intensity (a.u.) of the bands in the Raman spectra of GO, rGO, and CuO/rGO Hybrid.

\begin{tabular}{ccccc}
\hline Sample & D Band & G Band & 2D band & D+G \\
\hline GO & 1877 & 1947 & 181 & 273 \\
rGO & 321 & 265 & 48 & 32 \\
\hline
\end{tabular}




\begin{tabular}{lllll}
\hline $\mathrm{CuO} / \mathrm{rGO}$ & 411 & 305 & 41 & 43 \\
\hline
\end{tabular}

Table 2. FWHM $\left(\mathrm{cm}^{-1}\right)$ values of the bands in Raman spectra of GO, $\mathrm{rGO}$, and $\mathrm{CuO} / \mathrm{rGO}$ Hybrid.

\begin{tabular}{ccccc}
\hline Sample & D Band & G Band & 2D band & D+G \\
\hline $\mathrm{GO}$ & 153 & 75 & 241 & 350 \\
$\mathrm{rGO}$ & 76 & 288 & 82 & 82 \\
$\mathrm{CuO} / \mathrm{rGO}$ & 81 & 60 & 146 & 120 \\
\hline
\end{tabular}

\subsection{SEM and TEM Analysis}

The structure and morphology of the synthesized materials can be studied using SEM and TEM. Figure 5(a) shows the SEM image of rGO, which consists of wrinkles on its surface. Figure $5(\mathrm{~b})$ shows the surface of the $\mathrm{CuO} / \mathrm{rGO}$ hybrid, wherein the $\mathrm{CuO}$ NPs are distributed throughout the graphene sheets.
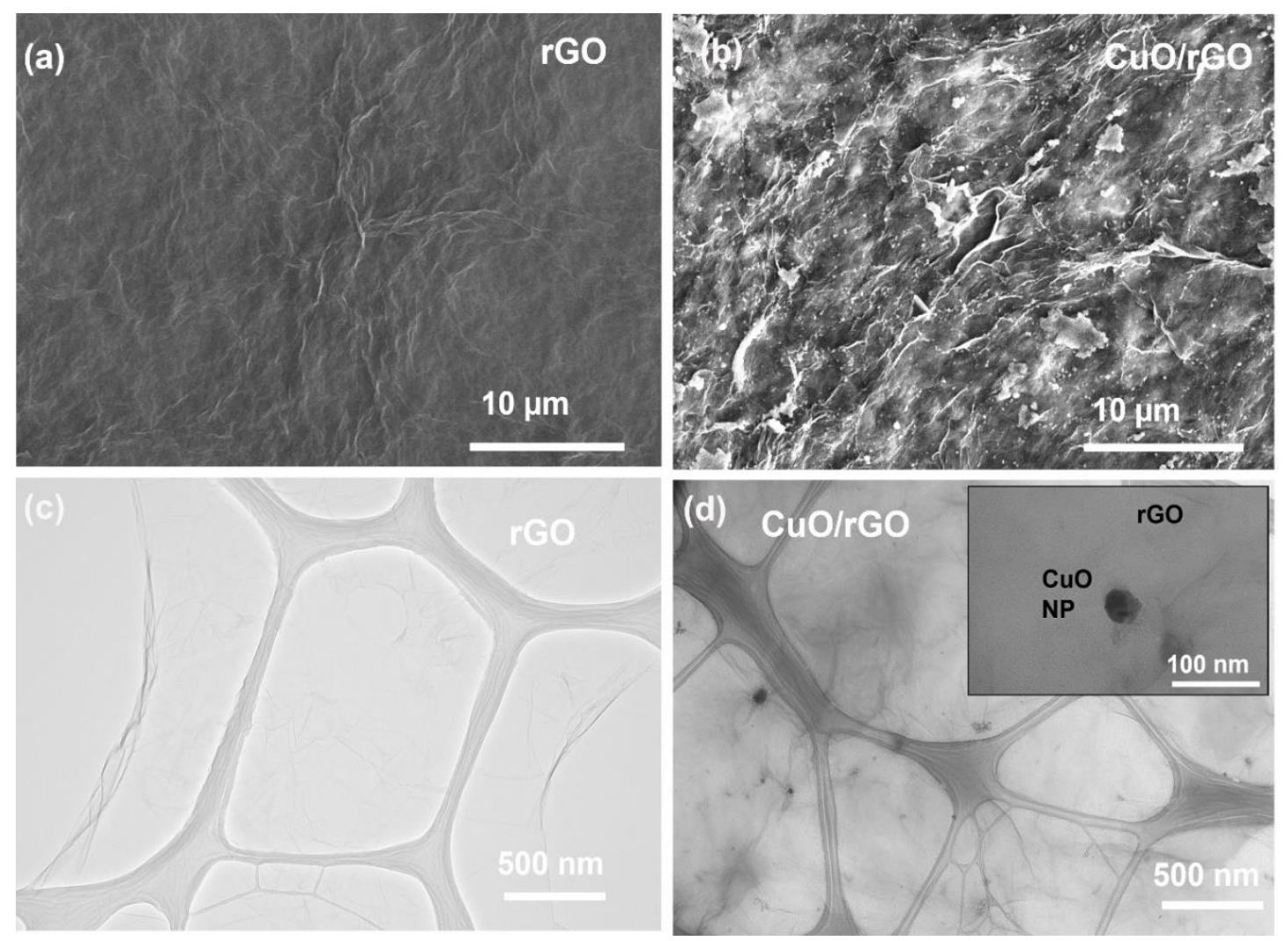

Figure 5. SEM image of (a) rGO and (b) $\mathrm{CuO} / \mathrm{rGO}$ hybrid, TEM images of (c) rGO and (d) $\mathrm{CuO} / \mathrm{rGO}$ hybrid. A high-resolution image of the hybrid is shown in the inset.

Figure 5(c) shows the TEM-based rGO morphology. The surface of rGO was identified as a continuous thin layer with few folds and wrinkles on the edges of rGO sheets. Figure $5(\mathrm{~d})$ shows the surface morphology of the $\mathrm{CuO} / \mathrm{rGO}$ hybrid wherein, the $\mathrm{CuO}$ in the form of nanoparticles were well-mixed with rGO. The size (diameter) of $\mathrm{CuO}$ particles was observed in the range of $10-40 \mathrm{~nm}$. The $\mathrm{CuO}$ nanoparticles were observed 
to be randomly distributed on the basal plane of rGO sheets, providing enough surface contact for charge transport. The inset shows the high-resolution image of the $\mathrm{CuO} / \mathrm{rGO}$ hybrid, displaying the $35 \mathrm{~nm} \mathrm{CuO}$ nanoparticles on the rGO sheets.

\section{a. Electrical Analysis}

Figure 6 shows the sheet resistance $(R s)$ of the $\mathrm{rGO}$ and $\mathrm{CuO} / \mathrm{rGO}$ hybrid thin films. To investigate the electrical properties of materials four-point probe technique has been used. The electrical characterization is carried out at room temperature. Rs of rGO and $\mathrm{CuO} / \mathrm{rGO}$ hybrid was found to be $1.85 \pm 0.12 \mathrm{k} \Omega / \square$ and $1.665 \pm 0.11 \mathrm{k} \Omega / \square$, respectively. The lower sheet resistance of hybrid is probably due to the presence of $\mathrm{CuO}$ nanoparticles.

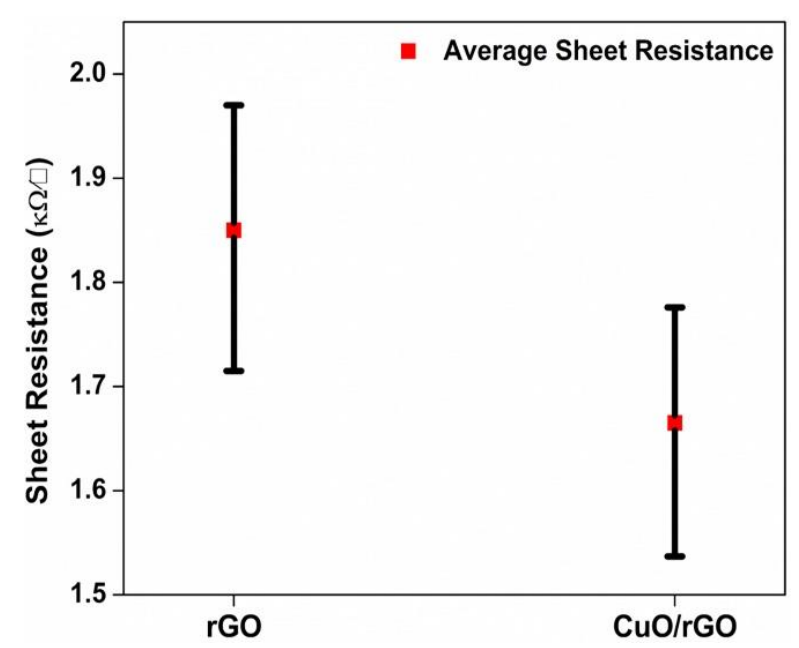

Figure 6. The sheet resistance of $\mathrm{rGO}$, and $\mathrm{CuO} / \mathrm{rGO}$ hybrid thin films.

\subsection{Gas Sensing Performance}

Figure $7(\mathrm{a})$ shows the response and recovery curve of $\mathrm{rGO} / \mathrm{CuO}$ hybrid thin film-based QCM gas sensors for $500 \mathrm{ppm} \mathrm{CO}_{2}$ at room temperature. A frequency shift $(\Delta \mathrm{f})$ of $438 \mathrm{~Hz}$ was shown by the gas sensor with $\mathrm{rGO} / \mathrm{CuO}$ hybrid sensing thin-film whereas, $\Delta \mathrm{f}$ of $193 \mathrm{~Hz}$ was demonstrated by rGO thin film-based sensor. The higher response of hybrid sensing film is possibly due to the presence of more OFGs at its surface as indicated by FTIR spectra. The $\mathrm{CO}_{2}$ gas was also exposed to GO coated QCM sensor at RT. The prepared GO coated QCM sensor showed no response upon $\mathrm{CO}_{2}$ gas exposure possibly due to the unavailability of active sites on its surface. GO has mainly hydroxyl and epoxide groups at its basal plane and it loses $\mathrm{sp}^{2}$ hybridization during the oxidation process which makes GO an insulating material at room temperature [35].

The comparison between response time, recovery time, and sensitivity of rGO and $\mathrm{CuO} / \mathrm{rGO}$ hybrid-based gas sensors is presented in Figure 7(b). The response time ( $\left.T_{\text {res }}\right)$ was observed to be $43 \mathrm{~s}$ and $48 \mathrm{~s}$ for $\mathrm{CuO} / \mathrm{rGO}$ hybrid and rGO thin film-based sensors, respectively, for 500 ppm $\mathrm{CO}_{2}$ gas at RT. The recovery time of hybrid and rGO-based sensors was observed to be $24 \mathrm{~s}$ and $21 \mathrm{~s}$, respectively. The $\mathrm{CuO} / \mathrm{rGO}$ hybrid-based sensor showed more recovery time than that of the rGO-based sensor. It is possibly due 
to the formation of hydroxyl groups. The gas sensing characteristics, frequency shift, response time, and recovery time strongly depend on the interactions between the oxygen functional group (OFGs) and analyte gas molecule [12,48]. The hybrid requires a higher time to recover due to the formation of hydroxyl bonds. Hydroxyl bonds are mainly attached to the basal plane however carboxyl and carbonyls are mainly attached to the edge plane of graphene [35]. These hydroxyls work as trap charges for the analyte gas molecule. Although the $\mathrm{rGO}$ and $\mathrm{CuO} / \mathrm{rGO}$ hybrid thin film-based sensors showed a good response towards $\mathrm{CO}_{2}$ gas, both sensors demonstrated baseline drift because the adsorbed $\mathrm{CO}_{2}$ molecules do not desorb completely during the desorption process. The calculated sensitivity of hybrid and rGO-based gas sensors was found to be $15 \mathrm{~Hz} / \mu \mathrm{g}$ and $1 \mathrm{~Hz} / \mu \mathrm{g}$ for $500 \mathrm{ppm}$ of $\mathrm{CO}_{2}$ gas.
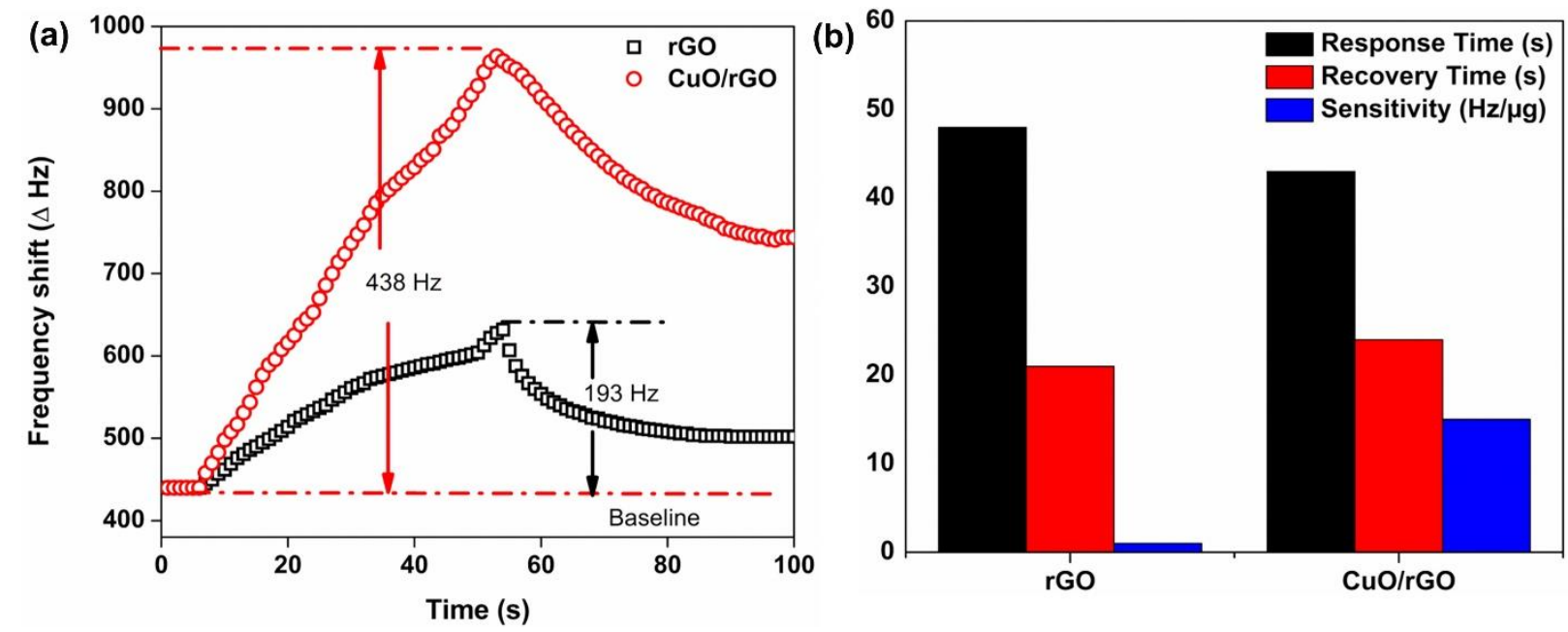

Figure 7. (a) Sensing response curve and (b) comparisons of response time (s), recovery time (s), and sensitivity $(\mathrm{Hz} / \mu \mathrm{g})$ of $\mathrm{rGO}$ and $\mathrm{CuO} / \mathrm{rGO}$ hybrid-based gas sensors.

\section{Conclusion}

In this work, we have successfully synthesized the $\mathrm{CuO} /$ functionalized graphene hybrid material. The thin films of material were developed by the drop-casting method on the substrates. The material properties were examined using advanced spectroscopy and microscopy. In hybrid nanostructure, a wrinkled and folded graphene surface with randomly distributed $\mathrm{CuO}$ NPs of size 10-40 nm was observed. The hybrid material was also found to have a large amount of OFGs and defective sites on its surface. The electrical conductivity of $\mathrm{CuO} / \mathrm{rGO}$ hybrid thin film was measured to be $\sim 1.5 \mathrm{k} \Omega / \square$. The functionality of the $\mathrm{CuO} / \mathrm{rGO}$ hybrid was investigated as a sensing layer in the QCM sensor for gas detection at room temperature. The $\mathrm{CuO} / \mathrm{rGO}$ hybrid-based gas sensor realized a high sensing response ( $\Delta \mathrm{f} \sim 440 \mathrm{~Hz}, T_{\text {res }} \sim 43 \mathrm{~s}$ ) for $500 \mathrm{ppm} \mathrm{CO}_{2}$ gas at room temperature. The sensing performance of the $\mathrm{CuO} / \mathrm{rGO}$ hybrid-based gas sensor was compared with that of the rGO-based sensor. A 2-fold improved sensing response was achieved in comparison to the rGO-based sensor. The selectivity of the $\mathrm{CuO} / \mathrm{rGO}$ hybrid 
will be investigated in our upcoming work. This study shows the interesting possibilities of $\mathrm{CuO} / \mathrm{rGO}$ hybrids in future gas sensing devices.

\begin{abstract}
Author Contributions: Conceptualization, M.G. and H.F.H.; methodology, M.G. and P.K.; software, M.G.; formal analysis, M.G. and P.K.; investigation, M.G. and P.K.; resources, H.F.H.; data curation, M.G.; writing-original draft preparation, M.G.; writing-review and editing, P.K., and H.F.H.; visualization, M.G., P.K., and H.F.H.; supervision, H.F.H., and Z.A.B.; project administration, H.F.H.; funding acquisition, H.F.H. All authors have read and agreed to the published version of the manuscript.
\end{abstract}

Funding: This research was funded by Yayasan Universiti Teknologi PETRONAS (YUTP) grant (number: 015LC0-153). The publication charge was partially supported by the Center for Graduate Studies (CGS), UTP, Malaysia.

Data Availability Statement: The data are available upon request from the corresponding authors.

Acknowledgments: The authors would like to thank the graduate assistantship scheme from the CGS, UTP, Malaysia.

Conflicts of Interest: The authors declare no conflict of interest.

\title{
References
}

1. Leghrib, R. Design, Fabrication and Characterisation of Gas Sensors Based on Nanohybrid Materials, 2011.

2. Azuma, K.; Kagi, N.; Yanagi, U.; Osawa, H. Effects of low-level inhalation exposure to carbon dioxide in indoor environments: A short review on human health and psychomotor performance. Environ. Int. 2018, 121, 51-56.

3. Yu, B.F.; Hu, Z.B.; Liu, M.; Yang, H.L.; Kong, Q.X.; Liu, Y.H. Review of research on air-conditioning systems and indoor air quality control for human health. Int. J. Refrig. 2009, 32, 3-20, doi:10.1016/j.ijrefrig.2008.05.004.

4. Tanvir, N.B.; Yurchenko, O.; Wilbertz, C.; Urban, G. Investigation of CO2 reaction with copper oxide nanoparticles for room temperature gas sensing. J. Mater. Chem. A 2016, 4, 5294-5302, doi:10.1039/c5ta09089j.

5. Rathi, K.; Pal, K. Ruthenium decorated tungsten disulfide quantum dots for $\mathrm{CO} 2$ gas sensor. Nanot 2019, 31.

6. Daud, A.I.; Wahid, K.A.A.; Khairul, W.M. Room-temperature operated cyano-terminated ethynylated-thiourea as a resistive-type carbon dioxide (CO2) gas sensor. Org. Electron. 2019, 70, 32-41.

7. Young, S.-J.; Lin, Z.-D. Sensing Performance of Carbon Dioxide Gas Sensors with Carbon Nanotubes on Plastic Substrate. ECS J. Solid State Sci. Technol. 2017, 6, 72-74.

8. Cui, S.; Pu, H.; Wells, S.A.; Wen, Z.; Mao, S.; Chang, J.; Hersam, M.C.; Chen, J. Ultrahigh sensitivity and layer-dependent sensing performance of phosphorene-based gas sensors. Nat. Commun. 2015, 6, 1-9.

9. Zhang, M.; Brooks, L.L.; Chartuprayoon, N.; Bosze, W.; Choa, Y.H.; Myung, N. V. Palladium/single-walled carbon nanotube back-to-back schottky contact-based hydrogen sensors and their sensing mechanism. ACS Appl. Mater. Interfaces 2014, 6, 319326, doi:10.1021/am404328g.

10. Zia, A.I.; Syaifudin, A.R.M.; Mukhopadhyay, S.C.; Yu, P.L.; Al-Bahadly, I.H.; Gooneratne, C.P.; Kosel, J.; Liao, T.S. Electrochemical impedance spectroscopy based MEMS sensors for phthalates detection in water and juices. J. Phys. Conf. Ser. 2013, 439, doi:10.1088/1742-6596/439/1/012026.

11. Algamili, A.S.; Khir, M.H.M.; Dennis, J.O.; Ahmed, A.Y.; Alabsi, S.S.; Ba Hashwan, S.S.; Junaid, M.M. A Review of Actuation and Sensing Mechanisms in MEMS-Based Sensor Devices. Nanoscale Res. Lett. 2021, 16, doi:10.1186/s11671-021-03481-7.

12. Berouaken, M.; Talbi, L.; Alkama, R.; Sam, S.; Menari, H.; Chebout, K.; Manseri, A.; Boucheham, A.; Gabouze, N. Quartz Crystal Microbalance Coated with Vanadium Oxide Thin Film for CO 2 Gas Sensor at Room Temperature. Arab. J. Sci. Eng. 2018, 43, 5957-5963.

13. Yang, M.; He, J; Hu, X.; Yan, C.; Cheng, Z. CuO nanostructures as quartz crystal microbalance sensing layers for detection of trace hydrogen cyanide gas. Environ. Sci. Technol. 2011, 45, 6088-6094, doi:10.1021/es201121w.

14. Vashist, S.K.; Vashist, P. Recent advances in quartz crystal microbalance-based sensors. J. Sensors 2011, 2011.

15. Varga, M.; Laposa, A.; Kulha, P.; Kroutil, J.; Husak, M.; Kromka, A. Quartz crystal microbalance gas sensor with nanocrystalline diamond sensitive layer. Phys. Status Solidi Basic Res. 2015, 252, 2591-2597. 
16. Gupta, M.; Athirah, N.; Hawari, H.F. Graphene derivative coated QCM-based gas sensor for volatile organic compound (VOC) detection at room temperature. Indones. J. Electr. Eng. Comput. Sci. 2020, 18, 1279-1286, doi:10.11591/ijeecs.v18.i3.pp1279-1286.

17. Zhu, B.L.; Xie, C.S.; Wang, W.Y.; Huang, K.J.; Hu, J.H. Improvement in gas sensitivity of ZnO thick film to volatile organic compounds (VOCs) by adding TiO2. Mater. Lett. 2004, 58, 624-629, doi:10.1016/S0167-577X(03)00582-2.

18. Ren, H.; Gu, C.; Joo, S.W.; Zhao, J.; Sun, Y.; Huang, J. Effective hydrogen gas sensor based on NiO@rGO nanocomposite. Sensors Actuators B Chem. 2018, 266, 506-513, doi:10.1016/j.snb.2018.03.130.

19. Chen, W.; Zhou, Q.; Wan, F.; Gao, T. Gas sensing properties and mechanism of Nano-SnO2-based sensor for hydrogen and carbon monoxide. J. Nanomater. 2012, 2012, doi:10.1155/2012/612420.

20. Lee, Z.Y.; Hawari, H.F. Bin; Djaswadi, G.W. Bin; Kamarudin, K. A highly sensitive room temperature co2 gas sensor based on sno2-rgo hybrid composite. Materials (Basel). 2021, 14, 1-16, doi:10.3390/ma14030522.

21. Yuan, J.; Zhang, J.-J.; Yang, M.-P.; Meng, W.-J.; Wang, H.; Lu, J.-X. CuO Nanoparticles Supported on TiO2 with High Efficiency for CO2 Electrochemical Reduction to Ethanol. Catalysts 2018, 8, 171, doi:10.3390/catal8040171.

22. Bhuvaneshwari, S.; Gopalakrishnan, N. Facile synthesis of low dimensional $\mathrm{CuO}$ nanostructures and their gas sensing applications. Cryst. Res. Technol. 2016, 51, 145-153, doi:10.1002/crat.201500243.

23. Purushothaman, K.K.; Saravanakumar, B.; Babu, I.M.; Sethuraman, B.; Muralidharan, G. Nanostructured CuO/reduced graphene oxide composite for hybrid supercapacitors. RSC Adv. 2014, 4, 23485-23491, doi:10.1039/c4ra02107j.

24. Nagaraja, M.; Prashanth, S.; Pattar, J.; Mahesh, H.M.; Rajanna, K. Polyaniline-CuO nanocomposite: Electrical, structural and sensor properties. Mater. Today Proc. 2021, 1-4, doi:10.1016/j.matpr.2021.08.154.

25. Kumar, P.; Kang, C.H.; Burhanudin, Z.A.; Saheed, M.S.M.; Irshad, M.I.; Mohamed, N.M. Graphene-based hybrid thin films as transparent conductive electrode for optoelectronic devices. In Proceedings of the IEEE International Conference on Semiconductor Electronics, Proceedings, ICSE; 2016; Vol. 2016-Septe, pp. 216-219.

26. Mandayo, G.G.; González, F.; Rivas, I.; Ayerdi, I.; Herrán, J. BaTiO3-CuO sputtered thin film for carbon dioxide detection. Sensors Actuators, B Chem. 2006, 118, 305-310.

27. Yoon, H.J.; Jun, D.H.; Yang, J.H.; Zhou, Z.; Yang, S.S.; Cheng, M.M.C. Carbon dioxide gas sensor using a graphene sheet. Sensors Actuators, B Chem. 2011, 157, 310-313, doi:10.1016/j.snb.2011.03.035.

28. Macinnes, M.M.; Hlynchuk, S.; Acharya, S.; Lehnert, N.; Maldonado, S. Reduction of Graphene Oxide Thin Films by Cobaltocene and Decamethylcobaltocene. ACS Appl. Mater. Interfaces 2018, 10, 2004-2015, doi:10.1021/acsami.7b15599.

29. Lesiak, B.; Trykowski, G.; Tóth, J.; Biniak, S.; Kövér, L.; Rangam, N.; Stobinski, L.; Malolepszy, A. Chemical and structural properties of reduced graphene oxide-dependence on the reducing agent. J. Mater. Sci. 2021, 56, 3738-3754, doi:10.1007/s10853-020-05461-1.

30. Ayesh, A.I.; Ahmed, R.E.; Al-Rashid, M.A.; Alarrouqi, R.A.; Saleh, B.; Abdulrehman, T.; Haik, Y.; Al-Sulaiti, L.A. Selective gas sensors using graphene and CuO nanorods. Sensors Actuators, A Phys. 2018, 283, 107-112, doi:10.1016/j.sna.2018.09.068.

31. Guo, L.; Hao, Y.W.; Li, P.L.; Song, J.F.; Yang, R.Z.; Fu, X.Y.; Xie, S.Y.; Zhao, J.; Zhang, Y.L. Improved NO2 Gas Sensing Properties of Graphene Oxide Reduced by Two-beam-laser Interference. Sci. Rep. 2018, 8, 1-7.

32. Sharma, B.; Kim, J.S. MEMS based highly sensitive dual FET gas sensor using graphene decorated Pd-Ag alloy nanoparticles for H2 detection. Sci. Rep. 2018, 8, 1-9, doi:10.1038/s41598-018-24324-z.

33. Hu, N.; Yang, Z.; Wang, Y.; Zhang, L.; Wang, Y. Ultrafast and sensitive room temperature NH 3 gas sensors based on chemically reduced graphene oxide. 2013, doi:10.1088/0957-4484/25/2/025502.

34. Gupta, M.; Hawari, H.F.; Kumar, P.; Burhanudin, Z.A.; Tansu, N. Functionalized reduced graphene oxide thin films for ultrahigh co2 gas sensing performance at room temperature. Nanomaterials 2021, 11, 1-18, doi:10.3390/nano11030623.

35. Yang, H.; Li, J.S.; Zeng, X. Correlation between molecular structure and interfacial properties of edge or basal plane modified graphene oxide. ACS Appl. Nano Mater. 2018, 1, 2763-2773.

36. Kwan, Y.C.G.; Ng, G.M.; Huan, C.H.A. Identification of functional groups and determination of carboxyl formation temperature in graphene oxide using the XPS O 1s spectrum. Thin Solid Films 2015, 590, 40-48.

37. Zhang, D.; Jiang, C.; Liu, J.; Cao, Y. Investigation of CO2 reaction with copper oxide nanoparticles for room temperature gas sensing. Sensors Actuators B Chem. 2017, 247, 875-882, doi:10.1016/j.snb.2017.03.108.

38. Singh, P.; Nath, P.; Arun, R.K.; Mandal, S.; Chanda, N. Novel synthesis of a mixed Cu/CuO-reduced graphene oxide nanocomposite with enhanced peroxidase-like catalytic activity for easy detection of glutathione in solution and using a paper strip. RSC Adv. 2016, 6, 92729-92738.

39. Tang, Q.; Zhou, Z.; Chen, Z. Graphene-related nanomaterials: Tuning properties by functionalization. Nanoscale 2013, 5, 45414583, doi:10.1039/c3nr33218g.

40. Zhang, Q.; Pang, X.; Zhao, Y. Effect of the external velocity on the exfoliation properties of graphene from amorphous SiO2 surface. Crystals 2021, 11, doi:10.3390/cryst11040454.

41. Bhaumik, A.; Haque, A.; Taufique, M.; Karnati, P.; Patel, R.; Nath, M.; Ghosh, K. Reduced Graphene Oxide Thin Films with Very Large Charge Carrier Mobility Using Pulsed Laser Deposition. J. Mater. Sci. Eng. 2017, 06.

42. Li, Z.; Liu, Y.; Guo, D.; Guo, J.; Su, Y. Room-temperature synthesis of CuO/reduced graphene oxide nanohybrids for high-performance NO2 gas sensor. Sensors Actuators, B Chem. 2018, 271, 306-310, doi:10.1016/j.snb.2018.05.097.

43. Wu, J. Bin; Lin, M.L.; Cong, X.; Liu, H.N.; Tan, P.H. Raman spectroscopy of graphene-based materials and its applications in related devices. Chem. Soc. Rev. 2018, 47, 1822-1873, doi:10.1039/c6cs00915h.

44. Kumar, P.; Lin, K.; Seng, W.; Shuaib, M.; Saheed, M. Hybrid film of single-layer graphene and carbon nanotube as transparent conductive electrode for organic light emitting diode. Synth. Met. 2019, 257. 
45. Cheng, C.; Zhang, C.; Gao, X.; Zhuang, Z.; Du, C.; Chen, W. 3D Network and 2D Paper of Reduced Graphene Oxide/Cu2O Composite for Electrochemical Sensing of Hydrogen Peroxide. Anal. Chem. 2018, 90, 1983-1991, doi:10.1021/acs.analchem.7b04070.

46. Muzyka, R.; Drewniak, S.; Pustelny, T.; Chrubasik, M.; Gryglewicz, G. Characterization of graphite oxide and reduced graphene oxide obtained from different graphite precursors and oxidized by different methods using Raman spectroscopy. Materials (Basel). 2018, 11, 15-17, doi:10.3390/ma11071050.

47. Wu, S.; Wan, L.; Wei, L.; Talwar, D.N.; He, K.; Feng, Z. Temperature-dependent optical properties of graphene on Si and $\mathrm{SiO} 2 / \mathrm{Si}$ substrates. Crystals 2021, 11, doi:10.3390/cryst11040358.

48. Zaki, S.E.; Basyooni, M.A.; Shaban, M.; Rabia, M.; Eker, Y.R.; Attia, G.F.; Yilmaz, M.; Ahmed, A.M. Role of oxygen vacancies in vanadium oxide and oxygen functional groups in graphene oxide for room temperature CO2 gas sensors. Sensors Actuators, $A$ Phys. 2019, 294, 17-24. 